\title{
KPNB1 Gene
}

National Cancer Institute

\section{Source}

National Cancer Institute. KPNB1 Gene. NCI Thesaurus. Code C142197.

This gene is involved in nuclear import. 